\title{
Articles
}

\section{Monodisperse Magnetizable Composite Silica Spheres with Tunable Dipolar Interactions}

\author{
E. M. Claesson and A. P. Philipse* \\ Van't Hoff Laboratory for Physical and Colloid Chemistry, Debye Institute, Utrecht University, \\ Padualaan 8, 3584 CH Utrecht, The Netherlands
}

Received April 27, 2005. In Final Form: July 26, 2005

\begin{abstract}
We demonstrate that magnetic particles of maghemite $\left(\gamma-\mathrm{Fe}_{2} \mathrm{O}_{3}\right)$ and cobalt ferrite $\left(\mathrm{CoFe}_{2} \mathrm{O}_{4}\right)$ can be irreversibly attached to colloidal silica that is grafted with 3-mercaptopropyl(trimethoxy)silane (followed by the controlled growth of a silica layer) to obtain stable dispersions of monodisperse colloidal silica spheres that contain a dense shell of ferrite particles at an adjustable distance from the sphere surface. Magnetization of the ferrite shell induces a large dipole moment, and adjusting the thickness of the outer silica layer enables one to tune the contact attraction. This novel type of magnetizable silica colloid exhibits structure formation in a homogeneous field that varies from isotropic distributions to elongated dipolar chains.
\end{abstract}

\section{Introduction}

Magnetic particles can be used as colloidal model systems for studying dipole-dipole interactions and should, as such, consist of monodisperse particles with adjustable dipole moments. Dispersions of magnetic colloids of various ferrites that are prepared with a classic coprecipitation synthesis ${ }^{1-3}$ contain particles in the size range of $10-20 \mathrm{~nm}$ with a significant polydispersity both in size and in shape. Moreover, controlling the size during the coprecipitation is nearly impossible with this method, although a certain size fractionation can be carried out on the synthesized dispersion. ${ }^{4-6}$ With recently developed synthesis methods ${ }^{7}$ ferrite particles with a low polydispersity can be obtained with size control up to a diameter of $21 \mathrm{~nm} .{ }^{8}$ Coating single magnetic particles with a nonmagnetic layer of silica enables one to tune the dipoledipole interactions by controlling the thickness of the silica shell and thereby the distance of closest approach between the magnetic cores. ${ }^{9}$ A drawback of this system is the inherently weak magnetic interaction due to the small magnetic core volume: the decay of the dipoledipole interaction potential between single magnetic particles is such that even a silica shell of only a few nanometers screens these interactions, as further discussed in section II.

* Corresponding author. E-mail: A.P.Philipse@chem.uu.nl.

(1) Massart, R. IEEE Trans. Magn. 1981, 17, 1247-1249.

(2) Bee, A.; Massart, R.; Neveu, S. J. Magn. Magn. Mater. 1995, 149, $6-9$.

(3) Tourinho, F. A.; Franck, R.; Massart, R. J. Mater. Sci. 1990, 25, 3249-3254.

(4) Cabuil, V.; Massart, R.; Bacri, J.-C.; Perzynski, R.; Salin, D. J. Chem. Res., Synop. 1987, 130-131.

(5) Massart, R.; Dubois, E.; Cabuil, V.; Hasmonay, E. J. Magn. Magn. Mater. 1995, 149, 1-5.

(6) Lefebure, S.; Dubois, E.; Cabuil, V.; Neveu, S.; Massart, R. J. Mater. Res. 1998, 13, 2975-2981.

(7) Sun, S.; Zeng, H. J. Am. Chem. Soc. 2002, 124, 8204-8205.

(8) Klokkenburg, M.; Vonk, C.; Claesson, E. M.; Meeldijk, J. D.; Erné,

B. H.; Philipse, A. P. J. Am. Chem. Soc. 2004, 126, 16706-16707.

(9) Philipse, A. P.; van Bruggen, M. P. B.; Pathmamanoharan, C. Langmuir 1994, 10, 92-99.
Here we report on the synthesis of well-defined, monodisperse magnetic silica composite particles in the colloidal size range with a large induced dipole moment, such that the presence of a silica layer, in contrast to the particles described in the work of Philipse et al, ${ }^{9}$ still allows substantial magnetic attractions between the cores at contact. The well-known chemistry of silica makes it possible to incorporate fluorescent dye ${ }^{10}$ and modify the particle surface with functional groups. ${ }^{12,11}$ The singleparticle magnetic core in ref 9 is replaced with a magnetic composite particle consisting of many single-domain magnetic particles that are irreversibly attached to the surface of a silica core. When a homogeneous magnetic field is applied, a large magnetic dipole moment is induced in such composites as the dipole moments of the individual magnetic particles align with the field. The size of the silica core can easily be varied, which enables one to tune the maximum strength of the magnetic dipole moment, and varying the silica shell thickness allows one to tune the strength of the dipole-dipole interactions at particle contact.

With this possibility in mind, we investigated the preparation of silica spheres containing particles of hard magnetic cobalt ferrite $\left(\mathrm{CoFe}_{2} \mathrm{O}_{4}\right)$ and soft magnetic maghemite $\left(\gamma-\mathrm{Fe}_{2} \mathrm{O}_{3}\right)$ adsorbed on silica cores. The synthesis of the silica cores as well as the growth of a silica layer were carried out with the well-known Stöber method $^{13,14}$ in alkaline mixtures of ethanol and water. For the silica deposition step, it is therefore crucial that the silica-ferrite composite particles are stable at these conditions. Composites consisting of small magnetic particles adsorbed onto large spherical nonmagnetic

(10) van Blaaderen, A.; Vrij, A. Langmuir 1992, 8, 2921-2931. (11)

(12) van Helden, A. K.; Jansen, J.; Vrij, A. J. Colloid Interface Sci. 1981, 81, 354-368.

(13) Stöber, W.; Fink, A.; Bohn, E. J. Colloid Interface Sci. 1968, 26 $62-69$.

(14) Bogush, G. H.; Tracy, M. A.; Zukaski, C. F., IV J. Non-Cryst. Solids 1988, 104, 95-106. 
colloids have previously been achieved by mixing dispersions of oppositely charged particles. ${ }^{15,16}$ We investigated the possibility of adsorbing cobalt ferrite and maghemite onto silica particles by electrostatic attraction as well; however, we observed the desorption of magnetic particles in a wide range of $\mathrm{pH}$. Such desorption obstructs the formation of uniform silica-ferrite spheres with an outer layer of amorphous silica.

To avoid the desorption of magnetic particles during the growth of a silica shell, we investigated the attachment of magnetic particles to the silica surface via a silane coupling agent: a molecule with the general formula $\mathrm{R}_{n} \mathrm{SiX}_{(4-n)}$ that contains hydrolyzable groups, $\mathrm{X}$, which can react with the surface of the silica particle, and one nonhydrolyzable group, $\mathrm{R}$. This nonhydrolyzable group should contain a functional group that can bind to the surface of the maghemite and cobalt ferrite particles to link them to the silica surface. One possible group could, in this case, be a thiol group because thiols are known for having a high affinity for metallic surfaces. ${ }^{17,18}$ Thiolfunctionalized organic molecules have previously been used to bind cadmium sulfide particles ${ }^{19,20}$ and heavy metal ions ${ }^{21}$ to inorganic substrates, but, to our knowledge, this has not yet been applied to ferrite particles. We therefore studied whether the thiol-functionalized silane coupling agent 3-mercaptopropyl(trimethoxy)silane (MPTMS) could be used to bind maghemite and cobalt ferrite covalently to the surface of silica particles.

The size, polydispersity, and internal morphology of the synthesized composite particles were studied using scanning (SEM) and transmission (TEM) electron microscopy. The size and polydispersity of the silica cores were obtained with static light scattering (SLS) and TEM. The magnetic radii of the magnetite and cobalt ferrite particles were obtained from magnetization curves as well as TEM. The influence of the silica shell thickness on the magnitude of the dipolar contact attraction was studied by imaging the structure formation of silica-ferrite spheres in a homogeneous magnetic field.

\section{Interaction Model}

First, a simple model is introduced to estimate the magnetic dipole-dipole interaction between two silica spheres with embedded magnetic particles. Consider two single magnetic particles with a magnetic radius, $a_{\mathrm{m}}$, embedded inside silica spheres at a center-to-center distance of $r=2\left(a_{\mathrm{m}}+\Delta\right)$ (see Figure 1A). Here, $2 \Delta$ is the surface-to-surface distance between the two particles such that, at contact, $\Delta$ equals the thickness of the silica shell around the magnetic particles. Assuming there are two equal dipole moments, $\mu_{\mathrm{mp}}$, that are aligned in a headto-tail configuration, the interaction energy, $u(r)$, in units of $\mathrm{kT}$ can be written as

$$
\frac{u(r)}{\mathrm{kT}}=-2 \frac{\lambda}{r^{3}} r \geq 2 a_{\mathrm{m}}
$$

(15) Zhu, Y.; Da, H.; Yang, X.; Hu, Y. Colloids Surf., A 2003, 231, 123-129.

(16) Caruso, F. Adv. Mater. 2001, 13, 11-22

(17) Bain, C.; Troughton, E.; Tao, Y.-T.; Evall, J.; Whitesides, G.; Nuzzo, R. J. Am. Chem. Soc. 1989, 111, 321-335.

(18) Fauconnier, N.; Pons, J.; Roger, J.; Bee, A. J. Colloid Interface Sci. 1997, 194, 427-433.

(19) Hebalkar, N.; Kharrazi, S.; Ethiraj, A.; Urban, J.; Flink, R.; Kulkarni, S. K. J. Colloid Interface Sci. 2004, 278, 107-114.

(20) Cestari, A. R.; Airoldi, C. J. Colloid Interface Sci. 1997, 195 $338-342$

(21) Arakaki, L.; Filha, V. A.; Espinola, J.; da Fonseca, M.; de Oliveira, S.; Arakaki, T.; Airoldi, C. J. Environ. Monit. 2003, 5, 366-370.
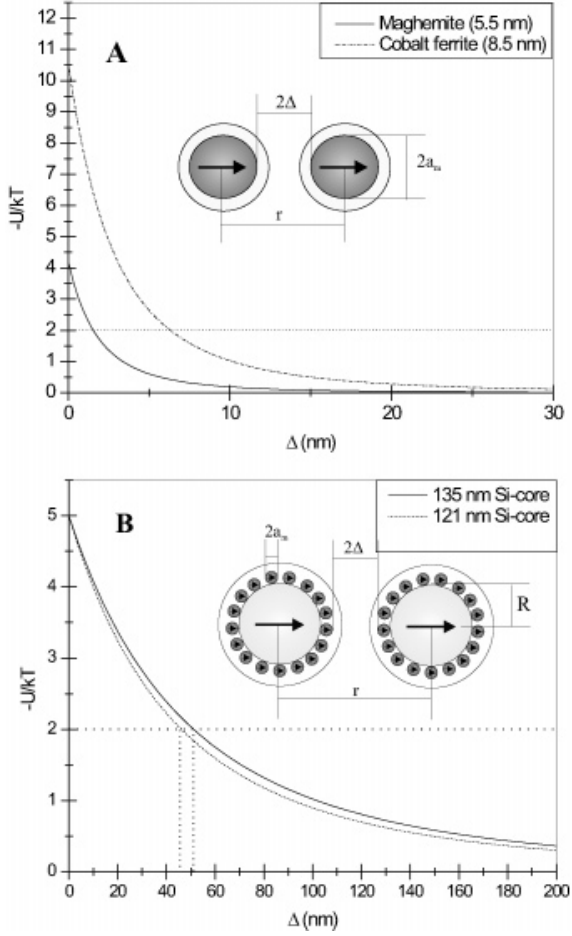

Figure 1. Interaction potentials for the interaction between two single magnetic particles of cobalt ferrite and of maghemite (A) and two composite particles with cobalt ferrite (B) as a function of the surface-to-surface distance, $\Delta$.

with the center-to-center distance $r=2\left(a_{\mathrm{m}}+\Delta\right)$. The interaction parameter $\lambda$ is a function of the magnetic moment of one magnetic particle:

$$
\lambda=\frac{\mu_{0} \mu_{\mathrm{mp}}{ }^{2}}{4 \pi \mathrm{kT}}=\frac{4}{9} \frac{\mu_{0} m_{\mathrm{s}}{ }^{2} \pi}{\mathrm{kT}} a_{\mathrm{m}}{ }^{6}
$$

in which

$$
a_{\mathrm{m}}=\sqrt[3]{\left(\frac{\chi_{\mathrm{i}}}{M_{\mathrm{s}}} \frac{9 \mathrm{kT}}{4 \pi \mu_{0} m_{\mathrm{s}}}\right)}
$$

Here, $\chi_{\mathrm{i}}$ is the initial susceptibility, $M_{\mathrm{s}}$ the measured saturation magnetization, and $\mu_{0}=4 \pi \times 10^{-7} \mathrm{H} / \mathrm{m}$ is the vacuum permeability. The parameters $\chi_{\mathrm{i}}$ and $M_{\mathrm{s}}$ can be obtained from a measured magnetization curve, $M(H)$. The bulk saturation magnetization per volume, $m_{\mathrm{s}}$, is a material constant, which, at $\mathrm{T}=298.15 \mathrm{~K}$, is $414 \mathrm{kA} / \mathrm{m}$ for maghemite and $425 \mathrm{kA} / \mathrm{m}$ for cobalt ferrite. ${ }^{22}$ Here, the bulk value for maghemite and cobalt ferrite is used for the magnetic particles. However, the saturation magnetization of the magnetic particles will be lowered because of the surface pinning of the spins at the particle surface. Consequently, using bulk saturation magnetization results in a slightly overestimated magnetic radius. The dipole moment of one spherical magnetic particle can be written as

$$
\mu_{\mathrm{mp}}=\frac{4 \pi a_{\mathrm{m}}^{3}}{3} m_{\mathrm{s}}=\frac{3 \mathrm{kT}}{4 \pi 10^{-7}} \frac{\chi_{\mathrm{i}}}{M_{\mathrm{s}}}
$$

In Figure 1A, the interaction potential from eq 1 is shown as a function of $\Delta$, using experimental values for the $a_{\mathrm{m}}$ of cobalt ferrite $\left(a_{\mathrm{m}}=8.4 \mathrm{~nm}\right)$ and maghemite $\left(a_{\mathrm{m}}=5.8\right.$

(22) Berkovski, B., Bashtovoy, V., Eds.; Magnetic Fluids and Applications Handbook; Begell House Inc.: New York, 1996. 
$\mathrm{nm}$ ), which were prepared with classic coprecipitation methods. ${ }^{1-3}$ Figure 1A shows that the dipole-dipole interaction between two single magnetic particles prepared with this method will in general not be strong enough $(-\mathrm{U} / \mathrm{kT}>2)$ to allow for the formation of dipolar chains for values of $\Delta$ larger than $2 \mathrm{~nm}$ for maghemite or $6 \mathrm{~nm}$ for cobalt ferrite. As a consequence, the thickness of a silica layer around these single ferrite particles should not be larger than a few nanometers to avoid total screening of the dipolar interactions. Such a thin silica layer, however, is experimentally difficult to obtain.

For composite particles, as shown in Figure 1B, the interaction energy depends on the number of magnetic particles, $N$, adsorbed per silica sphere. Assuming that the total magnetic moment of a composite particle, $\mu_{\mathrm{cp}}$, in a saturating magnetic field, is the sum of all individual magnetic moments $\left(\mu_{\mathrm{cp}}=\mathrm{N} \mu_{\mathrm{mp}}\right)$, the interaction energy $U(r)$ between two composite particles can be written as

$$
\frac{U(r)}{\mathrm{kT}}=N^{2} \frac{u(r)}{\mathrm{kT}} r \geq 2\left(R+2 a_{\mathrm{m}}\right)
$$

with the center-to-center distance $r$ now given by

$$
r=2\left(R+2 a_{\mathrm{m}}+\Delta\right)
$$

In Figure 1B, the interaction potential between either 121- or 135-nm silica cores are shown as a function of $\Delta$. In both cases, the number of adsorbed particles $N$ has been chosen such that $U=5 \mathrm{kT}$ when $\Delta=0$. That is, when cobalt ferrite particles $\left(a_{\mathrm{m}}=8.4 \mathrm{~nm}\right)$ are attached to silica spheres for which $R=121 \mathrm{~nm}, N=41$ and silica spheres for which $R=135 \mathrm{~nm}, N=48$, which, as will be shown later, are realistic numbers. Figure 1 predicts that the silica layer can be made considerably thicker around composite particles (provided that $N$ is large enough) than around single ferrite particles while still allowing the formation of dipolar chains. Although eqs 1 and 5 apply to a pair of spherical dipoles, they also provide a reasonable estimation for the interactions between one dipolar sphere and a chain of dipolar spheres. In the latter case, the attraction between the dipole and the chain at contact will be somewhat larger, although not more than $20 \%$ of the attraction between two dipolar spheres. ${ }^{23}$

\section{Experimental Section}

A. Materials. Tetraethyl orthosilicate (TEOS; Merck), ethanol (Merck), ammonia solution (Merck), iron(II) chloride tetrahydrate (Fluka), iron(III) chloride hexahydrate (Fluka), nitric acid (65\%, Merck), iron(III) nitrate nonahydrate (Merck), cobalt(II) chloride hexahydrate (Fluka), sodium hydroxide pellets (Acros), 3-mercaptopropyl(trimethoxy)silane (MPTMS; Fluka), and aqueous tetramethylammonium hydroxide (TMAH) solution (25\%, Aldrich) were used as received unless stated otherwise. Only demineralized water was used.

B. Silica Dispersions. Dispersions of silica particles were prepared, according to the Stöber method, ${ }^{13,14}$ by the hydrolysis and condensation polymerization of TEOS in a mixture of ethanol, water, and ammonia. Glassware for the Stöber synthesis was carefully cleaned with detergent, rinsed extensively with demineralized water, and dried prior to use. Distilled TEOS $(75 \mathrm{~mL})$ was rapidly added to a mixture of $1770 \mathrm{~mL}$ of distilled ethanol and $152 \mathrm{~mL}$ of ammonia (25\%) under the liquid surface via a funnel while the mixture was stirred vigorously. After a few minutes, the mixture became turbid white, indicating the formation of silica particles. The dispersion was labeled SiA. Two other silica dispersions ( $\mathrm{SiB}$ and $\mathrm{SiC}$ ) were previously prepared in the same way but with different ammonia concen-

(23) Philipse, A. P.; Maas, D. Langmuir 2002, 18, 9977-9984. trations. The particle size and polydispersity were obtained with TEM and SLS.

C. Maghemite Particles. Magnetite $\left(\mathrm{Fe}_{3} \mathrm{O}_{4}\right)$ particles were prepared in a coprecipitation step based on the procedure of Massart et al,1,2,24 followed by the subsequent oxidation to maghemite $\left(\gamma-\mathrm{Fe}_{2} \mathrm{O}_{3}\right)$. Iron chloride salts $\left(3.29 \mathrm{~g}\right.$ of $\mathrm{FeCl}_{2} \cdot 4 \mathrm{H}_{2} \mathrm{O}$ and $8.68 \mathrm{~g}$ of $\mathrm{FeCl}_{3} \cdot 6 \mathrm{H}_{2} \mathrm{O}$ ) were dissolved in $380 \mathrm{~mL}$ of water. Next, $25 \mathrm{~mL}$ of ammonia solution (25\%) was added rapidly to the iron chloride solution under vigorous stirring, upon which a black precipitate was formed. This black precipitate was collected with a permanent magnet under the reaction flask, and the supernatant was decanted. The sediment was redispersed in $40 \mathrm{~mL}$ of $2.0 \mathrm{M} \mathrm{HNO}_{3}$. The magnetite particles were thereafter oxidized to maghemite by adding $60 \mathrm{~mL}$ of $0.35 \mathrm{M} \mathrm{Fe}\left(\mathrm{NO}_{3}\right)_{3} \cdot 9 \mathrm{H}_{2} \mathrm{O}$ and letting the mixture reflux for $1 \mathrm{~h}$. After being cooled to room temperature, the maghemite particles were sedimented on a magnet and rinsed twice with $100 \mathrm{~mL}$ of $2.0 \mathrm{M} \mathrm{HNO}_{3}$ before redispersing them in $80 \mathrm{~mL}$ of water. The dispersion was labeled FFM3.

D. Cobalt Ferrite Particles. Cobalt ferrite $\left(\mathrm{CoFe}_{2} \mathrm{O}_{4}\right)$ was prepared by the coprecipitation of iron chloride salts and cobalt chloride salts, according to the procedure developed by Tourinho et $\mathrm{al}^{3}$ with some modifications. ${ }^{25}$ First, $2.38 \mathrm{~g}(0.01 \mathrm{~mol})$ of $\mathrm{CoCl}_{2}$. $6 \mathrm{H}_{2} \mathrm{O}$ was dissolved in a solution of $1 \mathrm{~mL}$ of $\mathrm{HCl}(37 \%)$ in $4 \mathrm{~mL}$ of water, and $5.406 \mathrm{~g}(0.02 \mathrm{~mol})$ of $\mathrm{FeCl}_{3} \cdot 6 \mathrm{H}_{2} \mathrm{O}$ was dissolved in $40 \mathrm{~mL}$ water. Second, the two separate solutions were both heated to $50{ }^{\circ} \mathrm{C}$ and thereafter mixed before being quickly added to 200 $\mathrm{mL}$ of boiling $1.0 \mathrm{M} \mathrm{NaOH}$ under vigorous stirring; a black precipitate was formed instantly, indicating the formation of cobalt ferrite particles. In the method of Tourinho et al, ${ }^{3}$ the boiling $\mathrm{NaOH}$-solution is poured into a boiling solution of the two metal salts, which prolongs the addition time (leading to a broader particle size distribution) and is less safe to handle. The reaction mixture was stirred for $30 \mathrm{~min}$ at $100{ }^{\circ} \mathrm{C}$ before being cooled to room temperature. The resulting black sediment was held to the glass wall with a magnet while the clear supernatant was decanted. In this way, the sediment was rinsed four times with $100 \mathrm{~mL}$ of water. After redispersing the sediment in 30 $\mathrm{mL}$ of $2.0 \mathrm{M} \mathrm{HNO}_{3}$ and stirring for $5 \mathrm{~min}, 30 \mathrm{~mL}$ of $0.35 \mathrm{M}$ $\mathrm{Fe}\left(\mathrm{NO}_{3}\right)_{3} \cdot 9 \mathrm{H}_{2} \mathrm{O}$ was added, and the mixture was refluxed for 45 min. Subsequently, the reaction mixture was cooled to room temperature and left to sediment on a magnet overnight. The resulting black, magnetic sediment was isolated by decanting the brownish, clear supernatant. The sediment was redispersed in water and labeled CoF12. The synthesis was repeated, and the new product was labeled $\mathrm{CoF} 13$.

E. Silica-Ferrite Composite Particles. 1. Electrostatic Attachment. To induce electrostatic attraction between negatively charged silica particles and positively charged ferrite particles, the silica particles were transferred from the Stöber mixture to an aqueous solution, and the $\mathrm{pH}$ was adjusted with nitric acid. When very little or no ferrite was adsorbed, a white layer of sedimented silica particles with a dark layer of ferrite particles on top was observed. The adsorption of ferrite particles was also monitored with TEM. For cobalt ferrite, a maximal attraction was found at $\mathrm{pH}=4.0-4.3$, and for maghemite, although this was not studied in great detail, it was found in the range of $\mathrm{pH}$ $=3-4$, as also observed by Smids and van Ewijk. ${ }^{26}$ Stable dispersions of silica and ferrite particles were mixed and shaken for $\sim 10 \mathrm{~min}$. After being mixed, the composite particles that were obtained flocculated immediately, and the sediment was rinsed with $\mathrm{HNO}_{3}$ solutions with appropriate $\mathrm{pH}$ values to maintain the electrostatic attraction while the nonadsorbed ferrite particles were being removed. A serious drawback of the electrostatic adsorption method was found to be the desorption of magnetic particles from the silica surface upon changing the $\mathrm{pH}$, a change that is necessary for the deposition of a silica layer onto the composite particles.

2. Chemical Attachment via a Silane Coupling Agent. To avoid the desorption of the magnetic particles, the silica particles were

(24) van Ewijk, G. Phase Behaviour of Mixtures of Magnetic Colloids and Nonadsorbing Polymers, Ph.D. Thesis, Utrecht University, 2001.

(25) Wagner, J.; Autenrieth, T.; Hempelmann, R. J. Magn. Magn. Mater. 2002, 252, 4-6.

(26) Smids, J.; van Ewijk, G. Ultrecht University, Utrecht, The Netherlands. Unpublished results, 2002. 
Table 1. Amounts of Chemicals Used for Grafting the Silica Particles with MPTMS

\begin{tabular}{ccccl}
\hline $\begin{array}{c}\text { sample code } \\
\text { silica dispersion } \\
\text { used) }\end{array}$ & $\begin{array}{c}V_{\text {Si-disp }}(\mathrm{mL}) \\
(\mathrm{mL})\end{array}$ & $\begin{array}{c}V_{\text {EtoH }} \\
(\mathrm{mL})\end{array}$ & $\begin{array}{c}V_{\text {NH3,29\% }} \\
\left(\mathrm{mol} \mathrm{MPTMS} / \mathrm{m}^{2} \mathrm{Si}\right)\end{array}$ \\
\hline SiM1 (SiC) & 20 & 10 & 0.5 & $0.0075\left(7.6 \times 10^{-6}\right)$ \\
SiM2 (SiC) & 20 & 10 & 0.5 & $0.075\left(7.6 \times 10^{-5}\right)$ \\
SiM4 (SiB) & 100 & 50 & 2.5 & $4.0\left(5.2 \times 10^{-4}\right)$ \\
SiM5 (SiB) & 100 & 50 & 2.5 & $0.4\left(5.2 \times 10^{-5}\right)$ \\
SiM6 (SiB) & 200 & 100 & 5.0 & $0.4\left(2.6 \times 10^{-4}\right)$ \\
SiM9 (SiA) & 200 & 100 & $a$ & $0.7\left(1.3 \times 10^{-4}\right)$ \\
SiM10 (SiA) & 200 & 100 & $a$ & $1.4\left(2.6 \times 10^{-4}\right)$ \\
SiM12 (SiA) & 200 & 100 & $a$ & $1.4\left(2.6 \times 10^{-4}\right)$
\end{tabular}

${ }^{a}$ Dispersion SiA was prepared $<4$ months before being grafted with MPTMS, and it was assumed that enough ammonia was still present.

first grafted with the thiol-functionalized silane coupling agent mercaptopropyl(trimethoxy)silane (MPTMS), which, as it turns out, irreversibly binds the magnetic particles to the silica surface. The methoxy groups of MPTMS are first hydrolyzed in the presence of ammonia, upon which oligomers are formed, which bind to the silanol groups on the surface of the silica particles through a condensation reaction. ${ }^{11,27}$

The MPTMS-coated silica was prepared as follows. The silica dispersions were diluted 1.5 times with ethanol. After this, ammonia solution (29\%) was added (in the case of more freshly prepared silica dispersions, no additional ammonia was needed) together with MPTMS while the mixture was being stirred (for synthesis details, see Table 1). After the mixture was stirred for approximately $45 \mathrm{~min}$, the temperature was raised to $80^{\circ} \mathrm{C}$, and approximately one-third of the total volume of the stable dispersion was slowly distilled at ambient pressure. After being cooled to room temperature, the particles were washed twice by sedimentation and redispersion in ethanol to remove any unreacted MPTMS. The silica particles were sedimented at 1500 rpm $(513 \mathrm{~g})$ using a Beckman-Coulter Spinchron DLX Centrifuge. Thereafter, the particles were transferred from ethanol to water by the same procedure, redispersing the sediment three times in water. The MPTMS-coated silica particles form stable dispersions in ethanol as well as in water. Ferrofluid of either maghemite or cobalt ferrite was added to the aqueous dispersion of MPTMS-coated silica particles, and the mixture was gently shaken for $\sim 1 \mathrm{~h}$ with an orbital shaker and subsequently left to settle without centrifugation. After adding cobalt ferrite to the first silica dispersion (SiM1) the sample was divided into two parts. One-half was stirred for $5 \mathrm{~min}$, and the other half was stirred for several hours before purifying the mixtures from free cobalt ferrite. The sediments of composite particles that formed were washed several times with water to remove nonadsorbed maghemite or cobalt ferrite. The composite particles that were prepared in this way were dispersed in a mixture of ethanol and ammonia and ultrasonicated for 30 min to verify whether the magnetic particles would desorb under these conditions, which are similar to the conditions under which a silica layer is grown onto the particles.

The MPTMS-coated silica particles were coded as SiM followed by a batch number and a letter indicating whether maghemite or cobalt ferrite had been added (e.g., SiM10C).

F. Silica-Ferrite Composite Particles Coated with Silica. The Stöber method used for the synthesis of silica particles (see section III.B) was also used for precipitating a silica layer onto the composite particles. To prevent aggregation during the coating procedure, it was necessary to work with a diluted dispersion of composite particles that had been subjected to ultrasonication and mechanical stirring. In addition to this, to prevent the formation of new silica particles, the glassware was cleaned carefully as described in section III.B. Adding too much TEOS at once may result in the secondary nucleation of silica particles;consequently, TEOS was added, at first, in small portions with a pipet. In later coating experiments, the pipet was replaced by a Gilson peristaltic pump for the dropwise

(27) Plueddeman, E. P. Silane Coupling Agents; Plenum Press: New York, 1982
Table 2. Synthesis Details for Silica Deposition on the Composite Particles

\begin{tabular}{ccrc}
\hline & $\begin{array}{c}\text { amount of } \\
\text { composite particles } \\
(\mathrm{g})\end{array}$ & $\begin{array}{c}\text { TEOS } \\
\text { addition rate } \\
(\mathrm{mol} / \mathrm{h})\end{array}$ & base \\
\hline sample code & 0.107 & $4.5 \times 10^{-4}$ & $\mathrm{NH}_{3}$ \\
SiM5Cs & 0.158 & $4.5 \times 10^{-4}$ & TMAH \\
SiM6Cs & 0.096 & $4.5 \times 10^{-4}$ & TMAH \\
SiM9Ms & 0.196 & $2.25 \times 10^{-4}$ & TMAH \\
SiM10Cs & 0.110 & $2.25 \times 10^{-4}$ & $\mathrm{TMAH}^{-4}$ \\
SiM10Ms & 0.189 & $1.2 \times 10^{-4}$ & $\mathrm{NH}_{3}$ \\
SiM12Ms & & &
\end{tabular}

addition of TEOS ${ }^{28,29}$ until a desired silica shell thickness had been obtained. The growth of the silica layer was monitored with TEM.

In the first growth experiment, $0.02 \mathrm{~g}$ of dispersion SiM1C in $5 \mathrm{~mL}$ of water was added to a mixture of $90 \mathrm{~mL}$ of ethanol and $3.6 \mathrm{~mL}$ of $\mathrm{NH}_{3}$ (29\%). Five portions of $10 \mu \mathrm{L}$ of TEOS ( $1 \mathrm{vol} \%$ in ethanol) was added to this mixture with a pipet in 10-min intervals. The ultrasonication and stirring of the reaction mixture was continued for $4 \mathrm{~h}$ after the last addition of TEOS. After the mixture was centrifuged, the particles were again redispersed in a mixture of $5 \mathrm{~mL}$ of water, $90 \mathrm{~mL}$ of ethanol, and $3.6 \mathrm{~mL}$ of $\mathrm{NH}_{3}(29 \%)$. The addition and cleaning procedure was repeated four times, adding portions of (1) $5 \times 10 \mu \mathrm{L}$, (2) $5 \times 20 \mu \mathrm{L}$, (3) $3 \times 100 \mu \mathrm{L}$, and (4) $4 \times 100 \mu \mathrm{L}$ of TEOS ( 1 vol \% in ethanol).

In later experiments, aqueous dispersions $(20 \mathrm{~mL})$ of the silicamaghemite/cobalt ferrite composite particles were mixed with ethanol and a base with a total volume of $1000 \mathrm{~mL}$ in a $3000 \mathrm{~mL}$ round-bottom flask equipped with a mechanical stirrer (for synthesis details, see Table 2). The flask was inserted into a 25 ${ }^{\circ} \mathrm{C}$ ultrasonic bath. TEOS ( $1 \mathrm{vol} \%$ in ethanol) was added dropwise at a rate of $\sim 4.5 \times 10^{-4} \mathrm{~mol} \mathrm{TEOS} / \mathrm{h}$ by using a peristaltic pump. Prior to use, the PVC tubings of the pump were rinsed with 10 $\mathrm{mL}$ of pure ethanol to remove any impurities that might act as seeds for new silica particles. Nitrogen flow around the inlet of TEOS/ethanol into the reaction flask was applied to prevent polymerization inside the droplets before reaching the dispersion of composite particles.

The first coating attempts were performed using ammonia $(0.6 \mathrm{M})$ as the base. However, the evaporation of ammonia retards or may even stop the silica growth. Therefore, an attempt was made to use 0.01 vol \% TMAH ( $10 \mathrm{~mL}, 1$ vol \% in water added to $1000 \mathrm{~mL}$ of reaction mixture) in the reaction mixture instead of ammonia to avoid this problem. ${ }^{30}$ For silica growth on cobalt ferrite composites this approach worked well. However, in the case of maghemite composites, TMAH prohibits silica growth for reasons that are not yet clear. For silica growth on maghemite composites, ammonia was used again, but with a higher initial concentration of ammonia $(0.8 \mathrm{M})$ than that used in the first experiments.

The coating procedures were continued for several days. After stopping the TEOS addition, the ultrasonication and stirring of the suspension was maintained for at least $4 \mathrm{~h}$ to make sure that all of the TEOS had reacted to avoid the formation of "dumbbells". The silica-coated samples were labeled by adding an "s" to the name of the starting dispersion (e.g., SiM10Cs).

G. Characterization Methods. TEM was performed with a Philips Tecni12 microscope using formfar-coated grids on which a droplet of dilute particle dispersion had been placed and from which the solvent had been evaporated. For each dispersion of composite particles, three TEM grids were prepared: the first one was dried at room temperature, the second was exposed to a homogeneous magnetic field of $1.0-1.3 \mathrm{~T}$ between the poles of a Bruker electromagnet for 15 min before it was left to dry, and the third grid was left to dry completely in the magnetic field. Particle size and polydispersity were determined from the TEM pictures using the imaging software AnalySIS. Magnetization curves were recorded on a Micromag 2900 alternating gradient magnetometer (Princeton Measurements Cooperation). From these curves, the remanence magnetization/saturation magne-

(28) Giesche, H. J. Eur. Ceram. Soc. 1994, 14, 189-204.

(29) Giesche, H. J. Eur. Ceram. Soc. 1994, 14, 205-214.

(30) Philipse, A. P. Colloids Surf., A 1993, 80, 203-210. 
Table 3. Radii of Silica Dispersions

\begin{tabular}{ccc}
\hline sample code & $R_{\mathrm{TEM}}(\mathrm{nm})$ & $R_{\mathrm{SLS}}(\mathrm{nm})$ \\
\hline $\mathrm{SiA}$ & $135 \pm 9$ & 152 \\
$\mathrm{SiB}$ & $121 \pm 6$ & 155 \\
$\mathrm{SiC}$ & $173 \pm 7$ & 204
\end{tabular}

Table 4. Properties of the Maghemite and Cobalt Ferrite Dispersions

\begin{tabular}{ccccc}
\hline $\begin{array}{c}\text { sample } \\
\text { code }\end{array}$ & $\begin{array}{c}R_{\text {TEM }} \\
(\mathrm{nm})\end{array}$ & $\begin{array}{c}\text { polydispersity } \\
(\%)\end{array}$ & $\begin{array}{c}a_{\mathrm{m}} \\
(\mathrm{nm})\end{array}$ & $\begin{array}{c}\text { interaction } \\
\text { parameter }(\lambda)\end{array}$ \\
\hline FFM3 & 3.3 & 29 & 5.8 & $2.8 \times 10^{-24}$ \\
CoF12 & 8.1 & 34 & 9.6 & $6.0 \times 10^{-23}$ \\
CoF13 & 7.3 & 30 & 8.4 & $2.6 \times 10^{-23}$
\end{tabular}

tization ratio, $M_{\mathrm{r}} / M_{\mathrm{s}}$, was calculated as a measure of the hysteresis in the sample.

\section{Results \& Discussion}

A. Synthesis of Silica Dispersions. TEM pictures of the silica dispersions show spherical particles with a low polydispersity in all cases. Radii obtained from TEM pictures (see Table 3) are usually smaller than radii obtained with SLS, which is due to the polydispersity and, in particular, the shrinkage of the particles due to the vacuum applied in the electron microscope.

B. Maghemite and Cobalt Ferrite Dispersions. The maghemite and cobalt ferrite particles are irregular in shape and polydisperse, which is common for particles prepared with a coprecipitation method. ${ }^{2,3}$ Estimates of the particle radii that were obtained from TEM pictures and magnetization curves are listed in Table 4.

C. Silica-Ferrite Composite Particles. 1. Electrostatic Attachment. Both the silica and the cobalt ferrite particles form stable dispersions in the $\mathrm{pH}$ range of 3.0-5.0. After mixing the silica and cobalt ferrite particles, the insufficient attraction between the silica and ferrite particles typically results in a white sediment (silica) and a dark (cobalt ferrite) supernatant. Furthermore, the occurrence of good adsorption, or lack thereof, can be observed with electron microscopy (see Figure 2). For cobalt ferrite, we found the maximum attraction at $\mathrm{pH}=$ 4.0-4.3, and for maghemite, the maximum attraction to the silica surface is expected to be in the range of $\mathrm{pH}=$ $3-4 .^{26}$ Because of this $\mathrm{pH}$ sensitivity, composite particles that are prepared with this method cannot be redispersed in a Stöber mixture for silica coating because it results in the desorption of the ferrite particles from the silica surface.

2. Chemical Attachment. Cobalt ferrite and maghemite can be attached to silica spheres coated with MPTMS without desorption when the $\mathrm{pH}$ or the solvent is changed. Compared to the composite particles prepared with the electrostatic adsorption method (see section IV.C.1), the layer of adsorbed ferrite particles is clearly more homogeneous and is usually a monolayer when the coupling agent is used. In Figure 4, TEM and SEM pictures of the dispersion SiM6 are shown after a very small amount of cobalt ferrite particles was added and after an additional larger amount of cobalt ferrite was added. In the first case, inhomogeneously covered silica particles are observed, but as more cobalt ferrite is added, the silica particles become fully covered with cobalt ferrite. However, an insufficient amount of the coupling agent on the surface of the silica particles results in little or even no cobalt ferrite/maghemite attaching to the silica particles, even after a large excess of magnetic particles were added to the silica dispersion. TEM studies also indicate that the time of stirring after mixing the silica and magnetic particle dispersions influences the amount of adsorbed

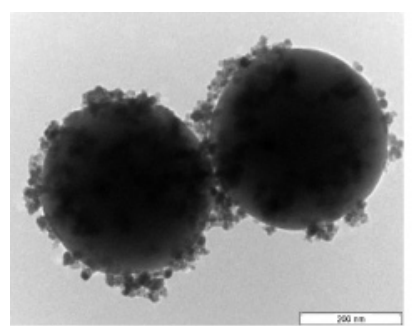

(a)

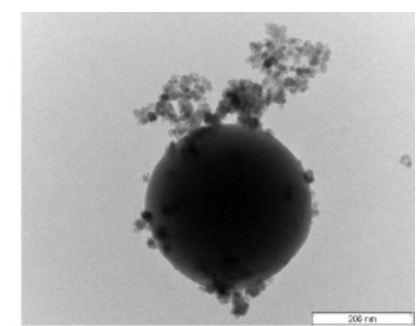

(b)
Figure 2. Adsorption of cobalt ferrite particles onto silica spheres via electrostatic attraction. The TEM pictures show the result of mixing the silica and cobalt ferrite dispersions at $\mathrm{pH}=4.0$ (a) and at $\mathrm{pH}=4.5(\mathrm{~b})$.
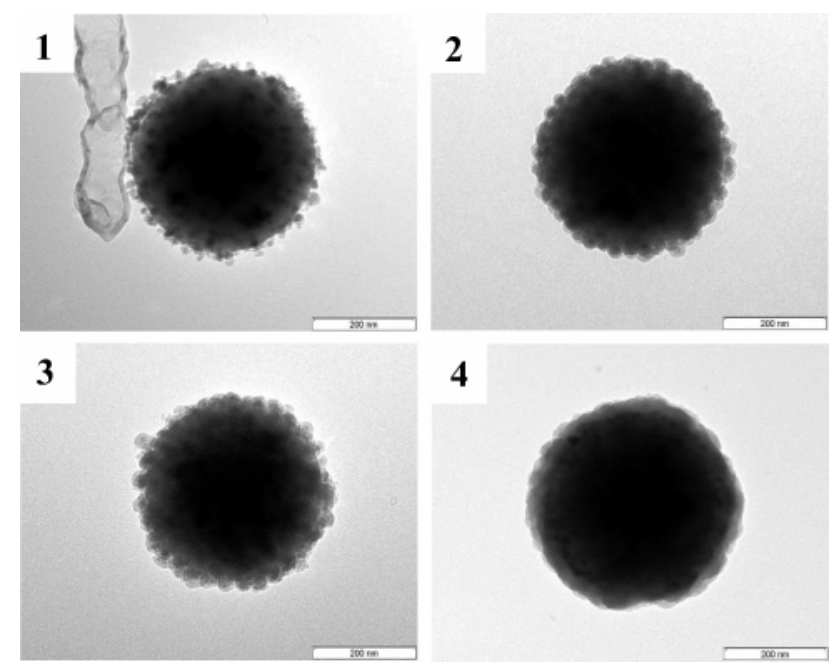

4

Figure 3. Silica growth onto composite particles of SiM2Co monitored by TEM, which shows the gradually increasing silica layer thickness from no silica present in picture 1 to a fully grown silica layer in picture 4 .

magnetic particles. Furthermore, it is observed that more MPTMS has to be used for maghemite than for cobalt ferrite particles to obtain silica particles fully covered with magnetic particles. At low concentrations of MPTMS, the silica surface can be fully covered with cobalt ferrite, whereas on the same surface, only a few maghemite particles can be attached.

D. Silica-Ferrite Composite Particles Coated with Silica. 1. Silica-Cobalt Ferrite Particles. The deposition of silica onto the silica-ferrite composite particles results in homogeneously coated particles with an irregular surface that smooths out as the silica layer grows (see Figures 3 and 5). The silica growth on SiM2Co took place in several steps, which allowed us to observe the growth of the silica layer (see Figure 3) using electron microscopy. However, growth in several steps, including purification between each step, inevitably results in the loss of particles, which reduces the yield of coated particles.

Using a peristaltic pump for the continuous addition of TEOS results in homogeneously coated particles. Although, with this coating method (which is continued for several days), the evaporation of ammonia during the growth eventually leads to a stop in the growth. This was observed for the dispersion SiM5C, in which only a thin silica layer precipitated on the particles, which was hardly visible with electron microscopy. The addition of more TEOS did not increase the thickness of the silica layer, nor had any new silica particles been formed. The silica growth on SiM6C and SiM10C shows that this problem can be solved by replacing the ammonia with TMAH, which 

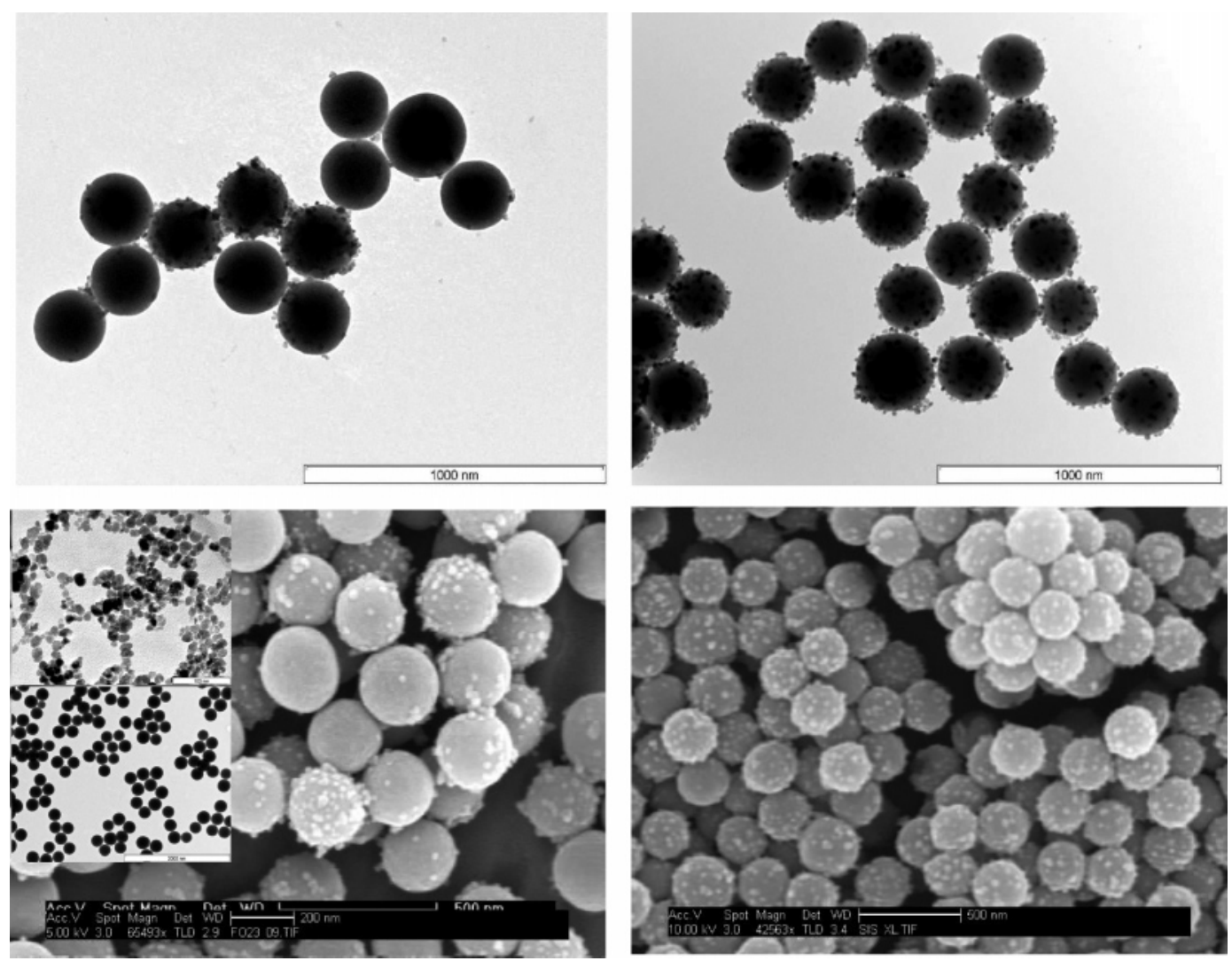

Figure 4. TEM and corresponding SEM picture of SiM6C after a small amount of cobalt ferrite had been added (left) and after an excess of cobalt ferrite had been added, followed by subsequent purification from free cobalt ferrite particles (right). The two insets show the starting particles CoF13 (top) and SiB (bottom).

does not evaporate and allows the silica growth to be continued for days without adding more TMAH.

The silica growth on the SiM6C composite particles resulted in a final radius of $200 \pm 10 \mathrm{~nm}$, and for SiM10Cs, the final radius was $168 \pm 9 \mathrm{~nm}$. This corresponds to silica layers of approximately 64 and $18 \mathrm{~nm}$, respectively. The cobalt ferrite particles are still visible through the silica layer in the electron microscope. Despite the dilution and ultrasonication, however, some aggregates are formed during the coating procedure, resulting in dumbbells and larger clusters coated with silica. Free cobalt ferrite particles were present in the reaction mixture as well, acting as seeds for silica growth. Purification of the dispersions is possible, but this inevitably leads to a loss of material. With a silica layer present, the composite particles become stable in ethanol as well as in water and DMSO. For example, the sedimentation rate of SiM6Cs in ethanol is $0.4 \mathrm{~mm} / \mathrm{h}$ compared to the starting dispersion, which flocculates immediately.

2. Silica-Maghemite Particles. In the first two attempts to grow a silica shell around the particles with maghemite, (SiM9M and SiM10M) TMAH was used. However, the TEM pictures showed that silica is formed but is not depositing on the core-shell particles. Instead, a large amount of new silica particles are formed. However, when using ammonia with a higher concentration than that used in the initial experiments (SiM5C), enough silica deposits before too much ammonia has evaporated. The reason for this difference between maghemite and cobalt ferrite is currently being investigated. The successfully coated particles of SiM12Ms, with a silica layer thickness of roughly $15 \mathrm{~nm}$, are shown in Figure 5 .

3. Magnetic Properties. TEM pictures of the dispersions that were dried either in a homogeneous magnetic field

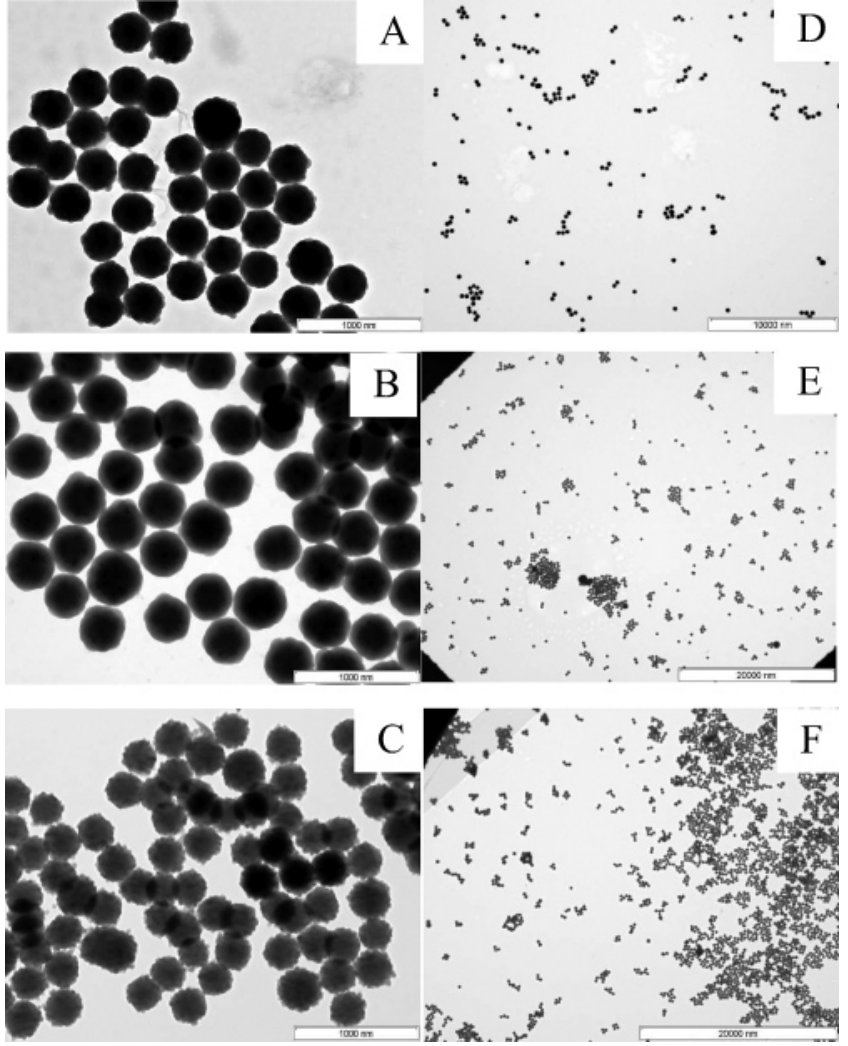

Figure 5. Dispersions of silica-coated composite particles SiM10Cs (A and D), SiM6Cs (B and E), and SiM12Ms (C and F). The dispersions were dried on the TEM grids without being exposed to a magnetic field. 


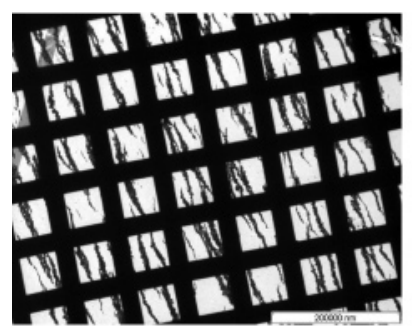

(a)

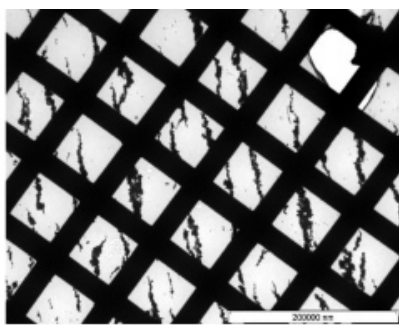

(b)

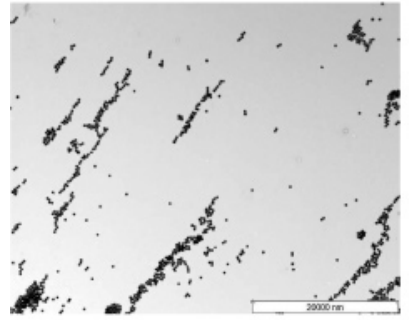

(c)

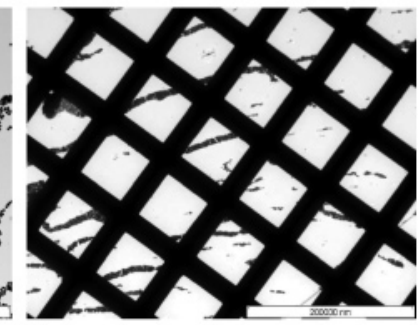

(d)

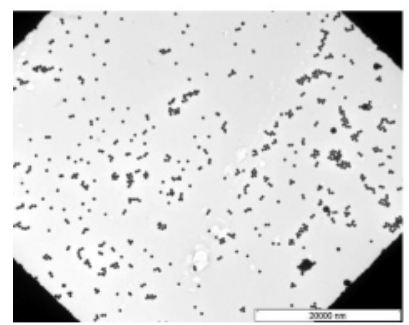

(e)

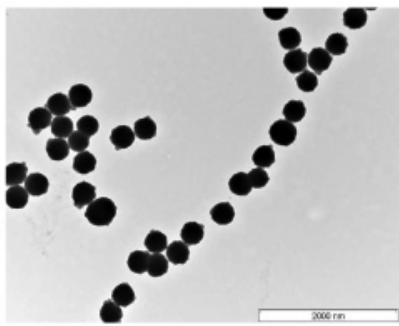

(f)

Figure 6. Dispersions of composite particles dried on a TEM grid in a homogeneous magnetic field. (a) SiM10C without silica coating; (b) SiM12M without silica coating; (c) SiM10Cs with an 18-nm silica layer; (d) SiM12Ms with a 15-nm silica layer; (e) SiM6Cs with a 64-nm silica layer; (f) magnified TEM picture of SiM10Cs. It can clearly be seen how the presence of a silica layer screens the dipole moments of the magnetic composite particles and how the extensive anisotropic structures of noncoated particles gradually disappears as the thickness of the silica layer increases.

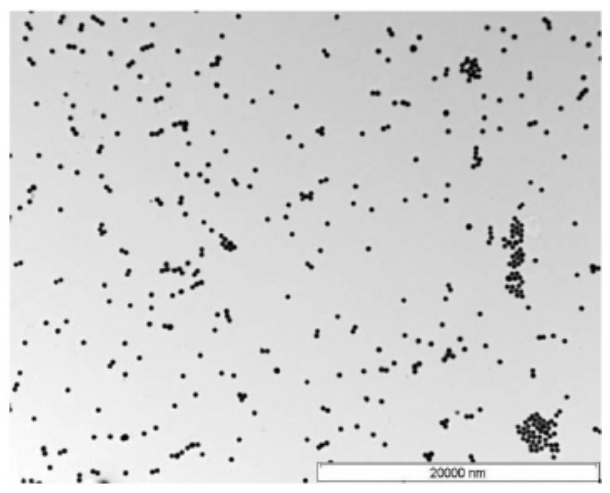

Figure 7. Dispersion of composite particles with SiM10Cs dried on a TEM grid after a 15-min exposure to a homogeneous magnetic field. The structures of the SiM10Cs particles formed in the homogeneous magnetic field (see Figure 6c) are not preserved after switching off the field on the time-scale of drying.

or after being exposed to a homogeneous magnetic field of $1.0-1.3 \mathrm{~T}$ for $15 \mathrm{~min}$ were compared to the pictures of the dispersions that had not been exposed to a field (see Figures 5-7).

The composites without silica coatings, with both cobalt ferrite and maghemite, all form extensive, anisotropic

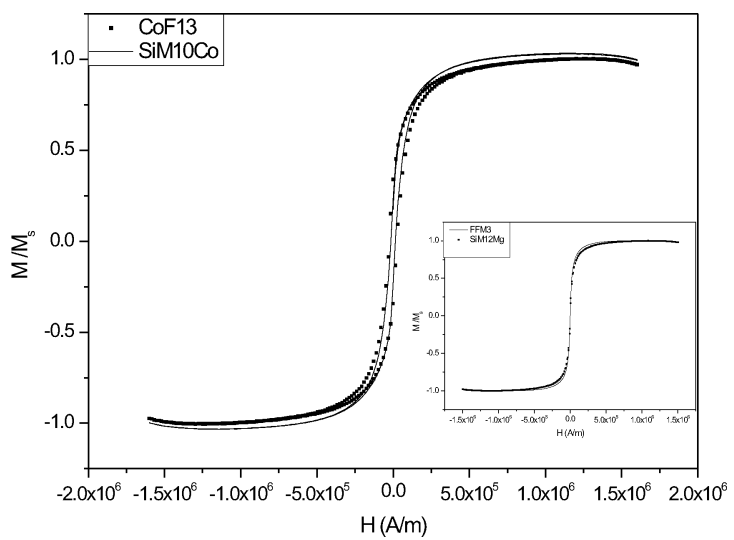

Figure 8. Magnetization curves of $\mathrm{CoF} 13$ and SiM10C together with the magnetization curves of FFM3 and SiM12M (inset).

Table 5. Remanence Magnetization/Saturation Magnetization from Magnetization Curves

\begin{tabular}{cc}
\hline sample code & $M_{\mathrm{r}} / M_{\mathrm{s}}$ \\
\hline FFM3 & 0.011 \\
CoF13 & 0.293 \\
SiM12M & 0.007 \\
SiM10C & 0.236 \\
SiM10C & $a$
\end{tabular}

${ }^{a}$ The sample was left for $1 \mathrm{~h}$ at $2 \mathrm{~T}$ before the magnetization curve was measured.

structures, which align with the magnetic field. When a silica layer is present, there is a clear difference between the particles with a shell thickness of $18 \mathrm{~nm}$ (SiM10Cs), for which the structures align with the field when dried in a homogeneous magnetic field, and the particles with $64 \mathrm{~nm}$ shell thickness (SiM6Cs), for which no significant difference is observed whether the dispersion has been exposed to a field or not.

In all cases, when the dispersions have been exposed to a field for 15 min before being dried, no extensive structures were observed, which indicates that the large dipole moment induced by the external field either partially or completely decays after switching off the field. This finding is supported by the magnetization curves of dry samples of SiM10C, SiM12M, CoF13, and FFM3, which are shown in Figure 8 and Table 5. For dry SiM10C, $M_{\mathrm{r}} / M_{\mathrm{s}}=0.24$, which implies that only $24 \%$ of the individual moments stay aligned after the external field has been removed. This roughly means that the interaction potential between the particles, after switching off the field, is only $6 \%$ of the interaction potential when all the dipole moments are aligned with a field according to eq 1 . For the maghemite particles, this effect is even lower (see Table 5), which is expected from a soft magnetic material.

The calculated number of adsorbed magnetic particles per silica sphere corresponding to a dipolar interaction potential of $5 \mathrm{kT}$ (see section II) is roughly the same as the that found in the SEM pictures of SiM6C. Furthermore, the calculated decay of the interaction potential in Figure $1 \mathrm{~B}$ is in agreement with the observations made here for the silica-cobalt ferrite particles, in that anisotropic structures can still be observed for a silica layer thickness of $18 \mathrm{~nm}$ but not when the silica layer thickness is $64 \mathrm{~nm}$, which means that the interaction is higher than $2 \mathrm{kT}$ in the first case, and is lower than $2 \mathrm{kT}$ in the latter case.

\section{Conclusions}

We have shown that well-defined, monodisperse magnetic silica particles can be prepared by the irreversible adsorption of maghemite or cobalt ferrite particles onto 
colloidal silica spheres that have been grafted with the thiol-functionalized silane coupling agent MPTMS. We have also shown that it is possible to electrostatically adsorb ferrite particles onto silica particles, but the drawback of this method is that it is strongly $\mathrm{pH}$-sensitive and the magnetic particles desorb at alkaline $\mathrm{pH}$. This problem is avoided when silica-ferrite composites are prepared using the coupling agent method, which yields composite particles that can be subjected to a change in solvent, strongly alkaline media, and ultrasonication without the ferrite particles desorbing from the silica surface. Further, the silica-ferrite composite particles can be coated with silica by the application of the Stöber method, in which the composite particles are introduced as seeds in a seeded growth step. Aggregation of the composite particles during the growth can be avoided by using ultrasonication, and once a silica coating is present, the particles form stable dispersions in ethanol, water, and DMSO.

In a homogeneous magnetic field, a large magnetic dipole moment is induced inside the silica-ferrite composite particles, which leads to the formation of extensive anisotropic structures that align with the field. When these composite particles have been coated with a thin silica layer (18 nm), such structures can be observed. However, as the thickness of the silica layer is increased to $64 \mathrm{~nm}$, anisotropic structures can no longer be observed, which is in agreement with the interaction model from section II. The possibility of tuning the interactions by varying the thickness of the silica layer and/or the size of the silica core provides a suitable colloidal model system for future microscopy studies of dipolar structure formation.

Acknowledgment. This work was financially supported by OCÉ Technologies, Venlo, The Netherlands. We thank Judith Wijnhoven and Dominique Thies-Weesie for helpful discussions about silica coatings and coupling agents, Chantal Vonk for helping with the preparation of silica particles and for helpful advice on colloidal syntheses, Stefano Sacanna for making SEM pictures, and Hans Meeldijk for technical support at the electron microscopy facility. Gerard van Ewijk and Jilles Smids are acknowledged for their explorative work on the adsorption of ferrite particles onto silica spheres, which was the starting point of this work. Ben Erné is acknowledged for many helpful discussions and input throughout this work.

LA051127A 